\title{
STRUCTURES INDUCED BY ALEXANDROV FUZZY TOPOLOGIES
}

\author{
YONG CHAN KIM
}

\begin{abstract}
In this paper, we investigate the properties of Alexandrov fuzzy topologies and meet-join approximation operators. We study fuzzy preorder, Alexandrov topologies and meet-join approximation operators induced by Alexandrov fuzzy topologies. We give their examples.
\end{abstract}

\section{INTRODUCTION}

Hájek [2] introduced a complete residuated lattice which is an algebraic structure for many valued logic. Höhle [3] introduced $L$-fuzzy topologies and $L$-fuzzy interior operators on complete residuated lattices. Pawlak $[8,9]$ introduced rough set theory as a formal tool to deal with imprecision and uncertainty in data analysis. Radzikowska [10] developed fuzzy rough sets in complete residuated lattice. Bělohlávek [1] investigated information systems and decision rules in complete residuated lattices. Zhang $[6,7]$ introduced Alexandrov $L$-topologies induced by fuzzy rough sets. Kim [5] investigated the properties of Alexandrov topologies in complete residuated lattices.

In this paper, we investigate the properties of Alexandrov fuzzy topologies and meet-join approximation operators in a sense as Höhle [3]. We study fuzzy preorder, Alexandrov topologies and meet-join approximation operators induced by Alexandrov fuzzy topologies. We give their examples.

\section{Preliminaries}

Definition 2.1 ([1-3]). A structure $(L, \vee, \wedge, \odot, \rightarrow, \perp, \top)$ is called a complete residuated lattice iff it satisfies the following properties:

Received by the editors March 26, 2014. Accepted June 3, 2014.

2010 Mathematics Subject Classification. 03E72, 03G10, 06A15, 54F05.

Key words and phrases. complete residuated lattices, fuzzy preorder, meet-join approximation operators, Alexandrov (fuzzy) topologies. 
(L1) $(L, \vee, \wedge, \perp, \top)$ is a complete lattice where $\perp$ is the bottom element and $T$ is the top element;

(L2) $(L, \odot, \top)$ is a monoid;

(L3) It has an adjointness,i.e.

$$
x \leq y \rightarrow z \text { iff } x \odot y \leq z .
$$

An operator ${ }^{*}: L \rightarrow L$ defined by $a^{*}=a \rightarrow \perp$ is called strong negations if $a^{* *}=a$.

$$
\top_{x}(y)=\left\{\begin{array}{ll}
\top, & \text { if } y=x, \\
\perp, & \text { otherwise. }
\end{array} \top_{x}^{*}(y)= \begin{cases}\perp, & \text { if } y=x \\
\top, & \text { otherwise. }\end{cases}\right.
$$

In this paper, we assume that $\left(L, \vee, \wedge, \odot, \rightarrow,^{*}, \perp, \top\right)$ be a complete residuated lattice with a strong negation *

Definition $2.2([6,7])$. Let $X$ be a set. A function $e_{X}: X \times X \rightarrow L$ is called a fuzzy preorder if it satisfies the following conditions

(E1) reflexive if $e_{X}(x, x)=1$ for all $x \in X$,

(E2) transitive if $e_{X}(x, y) \odot e_{X}(y, z) \leq e_{X}(x, z)$, for all $x, y, z \in X$ '

Example 2.3. (1) We define a function $e_{L}: L \times L \rightarrow L$ as $e_{L}(x, y)=x \rightarrow y$. Then $e_{L}$ is a fuzzy preorder on $L$.

(2) We define a function $e_{L^{X}}: L^{X} \times L^{X} \rightarrow L$ as $e_{L^{X}}(A, B)=\bigwedge_{x \in X}(A(x) \rightarrow$ $B(x))$. Then $e_{L^{X}}$ is a fuzzy preorder from Lemma $2.4(9)$.

Lemma $2.4([1,2])$. Let $\left(L, \vee, \wedge, \odot, \rightarrow,{ }^{*}, \perp, \top\right)$ be a complete residuated lattice with a strong negation ${ }^{*}$. For each $x, y, z, x_{i}, y_{i} \in L$, the following properties hold.

(1) If $y \leq z$, then $x \odot y \leq x \odot z$.

(2) If $y \leq z$, then $x \rightarrow y \leq x \rightarrow z$ and $z \rightarrow x \leq y \rightarrow x$.

(3) $x \rightarrow y=\top$ iff $x \leq y$.

(4) $x \rightarrow \top=\top$ and $\top \rightarrow x=x$.

(5) $x \odot y \leq x \wedge y$.

(6) $x \odot\left(\bigvee_{i \in \Gamma} y_{i}\right)=\bigvee_{i \in \Gamma}\left(x \odot y_{i}\right)$ and $\left(\bigvee_{i \in \Gamma} x_{i}\right) \odot y=\bigvee_{i \in \Gamma}\left(x_{i} \odot y\right)$.

(7) $x \rightarrow\left(\bigwedge_{i \in \Gamma} y_{i}\right)=\bigwedge_{i \in \Gamma}\left(x \rightarrow y_{i}\right)$ and $\left(\bigvee_{i \in \Gamma} x_{i}\right) \rightarrow y=\bigwedge_{i \in \Gamma}\left(x_{i} \rightarrow y\right)$.

(8) $\bigvee_{i \in \Gamma} x_{i} \rightarrow \bigvee_{i \in \Gamma} y_{i} \geq \bigwedge_{i \in \Gamma}\left(x_{i} \rightarrow y_{i}\right)$ and $\bigwedge_{i \in \Gamma} x_{i} \rightarrow \bigwedge_{i \in \Gamma} y_{i} \geq \bigwedge_{i \in \Gamma}\left(x_{i} \rightarrow y_{i}\right)$.

(9) $(x \rightarrow y) \odot x \leq y$ and $(y \rightarrow z) \odot(x \rightarrow y) \leq(x \rightarrow z)$.

(10) $x \rightarrow y \leq(y \rightarrow z) \rightarrow(x \rightarrow z)$ and $x \rightarrow y \leq(z \rightarrow x) \rightarrow(z \rightarrow y)$.

(11) $\bigwedge_{i \in \Gamma} x_{i}^{*}=\left(\bigvee_{i \in \Gamma} x_{i}\right)^{*}$ and $\bigvee_{i \in \Gamma} x_{i}^{*}=\left(\bigwedge_{i \in \Gamma} x_{i}\right)^{*}$

(12) $(x \odot y) \rightarrow z=x \rightarrow(y \rightarrow z)=y \rightarrow(x \rightarrow z)$ and $(x \odot y)^{*}=x \rightarrow y^{*}$. 
(13) $x^{*} \rightarrow y^{*}=y \rightarrow x$ and $(x \rightarrow y)^{*}=x \odot y^{*}$.

(14) $y \rightarrow z \leq x \odot y \rightarrow x \odot z$.

Definition $2.5([5])$. A map $\mathcal{M}: L^{X} \rightarrow L^{Y}$ is called an meet-join approximation operator if it satisfies the following conditions, for all $A, A_{i} \in L^{X}$, and $\alpha \in L$,

(M1) $\mathcal{M}(\alpha \rightarrow A)=\alpha \odot \mathcal{M}(A)$, where $(\alpha \rightarrow A)(x)=\alpha \rightarrow A(x)$ for each $x \in X$,

(M2) $\mathcal{M}\left(\bigwedge_{i \in I} A_{i}\right)=\bigvee_{i \in I} \mathcal{M}\left(A_{i}\right)$

(M3) $A^{*} \leq \mathcal{M}(A)$

(M4) $\mathcal{M}\left(\mathcal{M}^{*}(A)\right) \leq \mathcal{M}(A)$.

Definition 2.6 ([4]). An operator $\mathbf{T}: L^{X} \rightarrow L$ is called an Alexandrov fuzzy topology on $X$ iff it satisfies the following conditions, for all $A, A_{i} \in L^{X}$, and $\alpha \in L$,

(T1) $\mathbf{T}\left(\alpha_{X}\right)=\top$, where $\alpha_{X}(x)=\alpha$ for each $x \in X$,

(T2) $\mathbf{T}\left(\bigwedge_{i \in \Gamma} A_{i}\right) \geq \bigwedge_{i \in \Gamma} \mathbf{T}\left(A_{i}\right)$ and $\mathbf{T}\left(\bigvee_{i \in \Gamma} A_{i}\right) \geq \bigwedge_{i \in \Gamma} \mathbf{T}\left(A_{i}\right)$,

(T3) $\mathbf{T}(\alpha \odot A) \geq \mathbf{T}(A)$, where $(\alpha \odot A)(x)=\alpha \odot A(x)$ for each $x \in X$,

(T4) $\mathbf{T}(\alpha \rightarrow A) \geq \mathbf{T}(A)$.

Definition $2.7([5])$. A subset $\tau \subset L^{X}$ is called an Alexandrov topology if it satisfies satisfies the following conditions.

(O1) $\alpha_{X} \in \tau$.

(O2) If $A_{i} \in \tau$ for $i \in \Gamma, \bigvee_{i \in \Gamma} A_{i}, \bigwedge_{i \in \Gamma} A_{i} \in \tau$.

(O3) $\alpha \odot A \in \tau$ for all $\alpha \in L$ and $A \in \tau$.

(O4) $\alpha \rightarrow A \in \tau$ for all $\alpha \in L$ and $A \in \tau$.

Remark 2.8. (1) If $\mathbf{T}: L^{X} \rightarrow L$ is an Alexandrov fuzzy topology. Define $\mathbf{T}^{*}(A)=$ $\mathbf{T}\left(A^{*}\right)$. Then $\mathbf{T}^{*}$ is an Alexandrov fuzzy topology.

(2) If $\mathbf{T}$ be an Alexandrov fuzzy topology on $X, \tau_{T}^{r}=\left\{A \in L^{X} \mid \mathbf{T}(A) \geq r\right\}$ is an Alexandrov topology on $X$ and $\tau_{T}^{r} \subset \tau_{T}^{s}$ for $s \leq r \in L$.

\section{Structures Induced By Alexandrov Fuzzy Topologies}

Theorem 3.1. If $\mathcal{M}$ is a meet-join approximation operator, then $\tau_{\mathcal{M}}=\left\{A \in L^{X} \mid\right.$ $\left.\mathcal{M}(A)=A^{*}\right\}$ is an Alexandrov topology on $X$.

Proof. (O1) Since $\top_{X} \leq \mathcal{M}\left(\perp_{X}\right)$ and $\mathcal{M}\left(\top_{X}\right)=\mathcal{M}\left(\perp_{X} \rightarrow A\right)=\perp_{X} \odot \mathcal{M}(A)=\perp$, $\perp_{X}=\mathcal{M}\left(\top_{X}\right)$ and $\top_{X}=\mathcal{M}\left(\perp_{X}\right)$. Then $\perp_{X}, \top_{X} \in \tau_{\mathcal{M}}$.

(O2) For $A_{i} \in \tau_{\mathcal{M}}$ for each $i \in \Gamma$, by (M2), $\mathcal{M}\left(\bigwedge_{i \in \Gamma} A_{i}\right)=\bigvee_{i \in \Gamma} \mathcal{M}\left(A_{i}\right)=$ $\bigvee_{i \in \Gamma} A_{i}^{*}$. So, $\bigwedge_{i \in \Gamma} A_{i} \in \tau_{\mathcal{M}}$. Since $\bigwedge_{i \in \Gamma} A_{i}^{*} \leq \mathcal{M}\left(\bigvee_{i \in \Gamma} A_{i}\right) \leq \bigwedge_{i \in \Gamma} \mathcal{M}\left(A_{i}\right)=$ $\bigwedge_{i \in \Gamma} A_{i}^{*}$, Thus, $\bigvee_{i \in \Gamma} A_{i} \in \tau_{\mathcal{M}}$. 
(O3) For $A \in \tau_{\mathcal{M}}$, since $\alpha \odot \mathcal{M}(\alpha \odot A)=\mathcal{M}(\alpha \rightarrow(\alpha \odot A)) \geq \mathcal{M}(A), \mathcal{M}(\alpha \odot A) \geq$ $\alpha \rightarrow \mathcal{M}(A)=(\alpha \odot A)^{*}$. Then $\alpha \odot A \in \tau_{\mathcal{M}}$.

(O4) For $A \in \tau_{\mathcal{M}}$, by $(\mathrm{M} 4), \mathcal{M}(\alpha \rightarrow A)=\alpha \odot \mathcal{M}(A)=\alpha \odot A^{*}$. Hence $\alpha \rightarrow A \in$ $\tau_{\mathcal{M}}$

Theorem 3.2. Let $\mathbf{T}$ be an Alexandorv fuzzy topology on $X$. Define

$$
\begin{aligned}
R_{T}^{r}(x, y) & =\bigwedge\left\{A(x) \rightarrow A(y) \mid \mathbf{T}(A) \geq r^{*}\right\} \\
R_{T}^{-r}(x, y) & =\bigwedge\left\{B(y) \rightarrow B(x) \mid \mathbf{T}(B) \geq r^{*}\right\} .
\end{aligned}
$$

We have the following properties.

(1) $R_{T}^{r}$ is a fuzzy preorder with $R_{T}^{r} \leq R_{T}^{s}$ for each $s \leq r$.

(2) $R_{T}^{-r}$ is a fuzzy preorder with $R_{T}^{-r} \leq R_{T}^{-s}$ for each $s \leq r$ and

$$
R_{T}^{-r}(x, y)=R_{T^{*}}^{r}(x, y) .
$$

(3) Define $\mathcal{M}_{R_{T}^{r}}: L^{X} \rightarrow L^{X}$ as follows

$$
\mathcal{M}_{R_{T}^{r}}(A)(y)=\bigvee_{x \in X}\left(A^{*}(x) \odot R_{T}^{r}(x, y)\right) .
$$

Then $\mathcal{M}_{R_{T}^{r}}$ is a meet-join approximation operator on $X$ with $\mathcal{M}_{R_{T}^{r}} \leq \mathcal{M}_{R_{T}^{s}}$ for each $s \leq r$.

(4) $\tau_{T}^{r *}=\tau_{\mathcal{M}_{R_{T^{*}}^{r}}}$.

(5) $\mathcal{M}_{R_{T}^{-r}}$ is a meet-join approximation operator on $X$ such that

$$
\mathcal{M}_{R_{T}^{-r}}(A)(y)=\bigvee_{x \in X}\left(A^{*}(x) \odot R_{T}^{-r}(x, y)\right)=\bigvee_{x \in X}\left(A^{*}(x) \odot R_{T^{*}}(x, y)\right)
$$

(6) $\left(\tau_{T}^{r *}\right)^{*}=\tau_{\mathcal{M}_{R_{T}^{r}}}$

(7) $\mathcal{M}_{R_{T^{*}}^{r}}(A)=\bigwedge\left\{A_{i} \mid A^{*} \leq A_{i}, \mathbf{T}\left(A_{i}\right) \geq r^{*}\right\}$ for all $A \in L^{X}$ and $r \in L$. Moreover, $R_{T}^{r}(x, y)=\mathcal{M}_{R_{T^{*}}^{r}}\left(\top_{x}\right)(y)$, for each $x, y \in X$.

(8) $\mathcal{M}_{R_{T}^{r}}(A)=\bigwedge\left\{A_{i} \mid A^{*} \leq A_{i}, \mathbf{T}^{*}\left(A_{i}\right) \geq r^{*}\right\}$ for all $A \in L^{X}$ and $r \in L$. Moreover, $R_{T}^{-r}(x, y)=R_{T^{*}}^{r}(x, y)=\mathcal{M}_{R_{T^{*}}^{r}}\left(\top_{x}\right)(y)$, for each $x, y \in X$.

(9) If $\mathcal{M}_{R_{T}^{r_{i}}}(A)=B$ for all $i \in \Gamma \neq \emptyset$, then $\mathcal{M}_{R_{T}^{s}}(A)=B$ with $s=\bigwedge_{i \in \Gamma} r_{i}$.

(10) If $\mathcal{M}_{R_{T}^{-r_{i}}}(A)=B$ for all $i \in \Gamma \neq \emptyset$, then $\mathcal{M}_{R_{T}^{-s}}(A)=B$ with $s=\bigwedge_{i \in \Gamma} r_{i}$.

Proof. (1) Since $\mathbf{T}(B) \geq r^{*}$ iff $B \in \tau_{T}^{r *}$, then $R_{T}^{r}(x, y)=\bigwedge_{B \in \tau_{T}^{r *}}(B(x) \rightarrow B(y))$. Since $R_{T}^{r}(x, x)=\bigwedge_{B \in \tau_{T}^{r *}}(B(x) \rightarrow B(x))=\top$ and

$$
\begin{aligned}
& R_{T}^{r}(x, y) \odot R_{T}^{r}(y, z)=\bigwedge_{B \in \tau_{T}^{r *}}(B(x) \rightarrow B(y)) \odot \bigwedge_{B \in \tau_{T}^{r *}}(B(y) \rightarrow B(z)) \\
& \leq \bigwedge_{B \in \tau_{T}^{r *}}(B(x) \rightarrow B(y)) \odot(B(y) \rightarrow B(z)) \\
& \leq \bigwedge_{B \in \tau_{T}^{r *}}(B(x) \rightarrow B(z))=R_{T}^{r}(x, y) .
\end{aligned}
$$


Hence $R_{T}^{r}$ is a fuzzy preorder.

For $s \leq r$, since $\mathbf{T}(B) \geq s^{*} \geq r^{*}$, we have $R_{T}^{r} \leq R_{T}^{s}$.

(2) By a similar method as (1), $R_{T}^{-r}$ is a fuzzy preorder. Moreover,

$$
\begin{aligned}
R_{T}^{-r}(x, y) & =\bigwedge\left\{B(y) \rightarrow B(x) \mid \mathbf{T}(B) \geq r^{*}\right\} \\
& =\bigwedge\left\{B^{*}(x) \rightarrow B^{*}(y) \mid \mathbf{T}\left(B^{*}\right)=\mathbf{T}^{*}(B) \geq r^{*}\right\} \\
& =R_{T^{*}}^{r}(x, y) .
\end{aligned}
$$

(3) $(\mathrm{M} 1) \mathcal{M}_{R_{T}^{r}}\left(\bigwedge_{i \in \Gamma} A_{i}\right)(y)=\bigvee_{x \in X}\left(\left(\bigwedge_{i \in \Gamma} A_{i}\right)^{*}(x) \odot R_{T}^{r}(x, y)\right)=\bigvee_{i \in \Gamma} \mathcal{M}_{R_{T}^{r}}\left(A_{i}\right)(y)$

(M2)

$$
\begin{aligned}
& \mathcal{M}_{R_{T}^{r}}(\alpha \rightarrow A)(y)=\bigvee_{x \in X}\left((\alpha \rightarrow A)^{*}(x) \odot R_{T}^{r}(x, y)\right) \\
& =\bigvee_{x \in X}\left(\alpha \odot\left(A^{*}(x) \odot R_{T}^{r}(x, y)\right)\right)=\alpha \odot \mathcal{M}_{R_{T}^{r}}(A)(y)
\end{aligned}
$$

$(\mathrm{M} 3) \mathcal{M}_{R_{T}^{r}}(A)(y)=\bigvee_{x \in X}\left(A^{*}(x) \odot R_{T}^{r}(x, y)\right) \geq A^{*}(y) \odot R_{T}^{r}(y, y)=A^{*}(y)$.

(M4)

$$
\begin{aligned}
\mathcal{M}_{R_{T}^{r}}\left(\mathcal{M}_{R_{T}^{r}}^{*}(A)\right)(x) & =\bigvee_{y \in X}\left(\mathcal{M}_{R_{T}^{r}}(A)(y) \odot R_{T}^{r}(y, x)\right) \\
& =\bigvee_{y \in X}\left(\bigvee_{z \in X}\left(A^{*}(z) \odot R_{T}^{r}(z, y)\right) \odot R_{T}^{r}(y, x)\right) \\
& =\bigvee_{z \in X}\left(A^{*}(z) \odot \bigvee_{y \in X}\left(R_{T}^{r}(z, y) \odot R_{T}^{r}(y, x)\right)\right) \\
& \leq \bigvee_{z \in X}\left(A^{*}(z) \odot R_{T}^{r}(z, x)\right) \\
& =\mathcal{M}_{R_{T}^{r}}(A)(x) .
\end{aligned}
$$

For $s \leq r$, since $R_{T}^{r} \leq R_{T}^{s}$, then $\mathcal{M}_{R_{T}^{r}} \leq \mathcal{M}_{R_{T}^{s}}$.

(4) Since $A \in \tau_{T}^{r *}$;i.e. $\mathbf{T}(A) \geq r^{*}, R_{T^{*}}^{r}(x, y) \odot A^{*}(x)=\bigwedge_{B \in \tau_{T^{*}}^{r *}}(B(x) \rightarrow B(y)) \odot$ $A^{*}(x) \leq\left(A^{*}(x) \rightarrow A^{*}(y)\right) \odot A^{*}(x) \leq A^{*}(y)$, by $\mathrm{M}(3), \mathcal{M}_{R_{T^{*}}^{r}}(A)=A^{*}$. So, $A \in$ $\tau_{\mathcal{M}_{R^{*}}^{r}}$. Thus $\tau_{T}^{r *} \subset \tau_{\mathcal{M}_{R_{T^{*}}^{r}}}$. Let $A \in \tau_{\mathcal{M}_{R_{T^{*}}^{r}}}$;i.e. Let $\mathcal{M}_{R_{T^{*}}^{r}}(A)=A^{*}$. Then

$$
\begin{aligned}
A & =\mathcal{M}_{R_{T^{*}}^{r}}^{*}(A)=\left(\bigvee_{y \in X}\left(A^{*}(y) \odot R_{T^{*}}^{r}(y,-)\right)\right)^{*} \\
& =\bigwedge_{y \in X}\left(A(y) \rightarrow \bigvee_{B \in T_{T^{*}}^{r *}}\left(B(y) \odot B^{*}\right)\right)
\end{aligned}
$$

Since $\bigvee_{B \in \tau_{T^{*}}^{r *}}\left(B(y) \odot B^{*}\right) \in \tau_{T}^{r *}$ and $\bigwedge_{y \in X}\left(A(y) \rightarrow \bigvee_{B \in \tau_{T^{*}}^{r *}}\left(B(y) \odot B^{*}\right)\right) \in \tau_{T}^{r *}$, we have $A \in \tau_{T}^{r *}$. Hence $\tau_{\mathcal{M}_{R^{*}}^{r}} \subset \tau_{T}^{r *}$.

(5) It is similarly proved as (4).

(6) Let $A \in\left(\tau_{T}^{r *}\right)^{*}$. Since $A \in \tau_{T}^{r *}$,

$$
\begin{aligned}
R_{T}^{r}(x, y) \odot A^{*}(x) & =\bigwedge_{B \in \tau_{T}^{r *}}(B(x) \rightarrow B(y)) \odot A^{*}(x) \\
& \leq\left(A^{*}(x) \rightarrow A^{*}(y)\right) \odot A^{*}(x) \leq A^{*}(y) .
\end{aligned}
$$

Hence $\mathcal{M}_{R_{T}^{r}}(A)=A^{*}$;i.e. $A \in \tau_{\mathcal{M}_{R_{T}^{r}}}$. Thus $\left(\tau_{T}^{r *}\right)^{*} \subset \tau_{\mathcal{M}_{R_{T}^{r}}}$.

Let $A \in \tau_{\mathcal{M}_{R_{T}^{r}}}$;i.e. $\mathcal{M}_{R_{T}^{r}}(A)=A^{*}$. Then

$$
\begin{aligned}
A & =\mathcal{M}_{R_{T}^{r}}^{*}(A)=\left(\bigvee_{y \in X}\left(A(y) \odot R_{T}^{r}(y,-)\right)\right)^{*} \\
& =\bigwedge_{y \in X}\left(A^{*}(y) \rightarrow \bigvee_{B \in \tau_{T}^{r *}}\left(B(y) \odot B^{*}\right)\right)
\end{aligned}
$$


Since $\bigvee_{B \in \tau_{T}^{r *}}\left(B(y) \odot B^{*}\right) \in\left(\tau_{T}^{r *}\right)^{*}$ and $\bigwedge_{y \in X}\left(A^{*}(y) \rightarrow \bigvee_{B \in \tau_{T}^{r *}}\left(B(y) \odot B^{*}\right)\right) \in\left(\tau_{T}^{r *}\right)^{*}$, we have $A \in\left(\tau_{T}^{r *}\right)^{*}$. Hence $\left(\tau_{T}^{r *}\right)^{*}=\tau_{\mathcal{M}_{R_{T}^{r}}}$.

(7) For each $A \in L^{X}$ with $A^{*} \leq A_{i}, \mathbf{T}\left(A_{i}\right) \geq r^{*}$, since $A_{i} \in \tau_{T}^{r *}=\tau_{\mathcal{M}_{R_{T^{*}}^{r}}}$, then

$$
\bigwedge_{i} A_{i} \leq \mathcal{M}_{R_{T^{*}}^{r}}\left(\bigvee_{i} A_{i}^{*}\right) \leq A_{i}=\mathcal{M}_{R_{T^{*}}^{r}}\left(A_{i}^{*}\right)
$$

So, $\mathcal{M}_{R_{T^{*}}^{r}}\left(\bigvee_{i} A_{i}^{*}\right)=\bigwedge_{i} A_{i}$. Since $A \geq \bigvee A_{i}^{*}$,

$$
\mathcal{M}_{R_{T^{*}}^{r}}(A) \leq \mathcal{M}_{R_{T^{*}}^{r}}\left(\bigvee_{i} A_{i}^{*}\right)=\bigwedge_{i} A_{i}=\bigwedge\left\{A_{i} \mid A^{*} \leq A_{i}, \mathbf{T}\left(A_{i}\right) \geq r^{*}\right\} .
$$

Since $\mathcal{M}_{R_{T^{*}}^{r}}\left(\mathcal{M}_{R_{T^{*}}^{r}}^{*}(A)\right)=\mathcal{M}_{R_{T^{*}}^{r}}(A) \geq A^{*}$ and $\mathcal{M}_{R_{T^{*}}^{r}}(A) \in \tau_{\mathcal{M}_{R_{T^{*}}}^{r}}=\tau_{T}^{r *}$. So, $\bigwedge\left\{A_{i} \mid A^{*} \leq A_{i}, \mathbf{T}\left(A_{i}\right) \geq r^{*}\right\} \leq \mathcal{M}_{R_{T^{*}}^{r}}(A)$. Hence $\bigwedge\left\{A_{i} \mid A^{*} \leq A_{i}, \mathbf{T}\left(A_{i}\right) \geq r^{*}\right\}=$ $\mathcal{M}_{R_{T^{*}}^{r}}(A)$ for all $A \in L^{X}$ and $r \in L$.

(8) It is proved in a similar way as (7).

(9) Let $\mathcal{M}_{R_{T}^{r_{i}}}(A)=B$ for all $i \in \Gamma \neq \emptyset$. Since

$$
\begin{aligned}
& \mathcal{M}_{R_{T}^{r_{i}}}(A)=\bigvee_{x \in X}\left(A^{*}(x) \odot R_{T}^{r_{i}}(x,-)\right) \\
& =\bigvee_{x \in X}\left(A^{*}(x) \odot \bigwedge_{D \in \tau_{T}^{r_{i}^{*}}}(D(x) \rightarrow D)\right) \in \tau_{T}^{r_{i}^{*}}
\end{aligned}
$$

$\mathbf{T}(B)=\mathbf{T}\left(\mathcal{M}_{R_{T}^{r_{i}}}(A)\right) \geq r_{i}^{*}$, then $\mathbf{T}(B) \geq \bigvee_{i \in \Gamma} r_{i}^{*}=\left(\bigwedge_{i \in \Gamma} r_{i}\right)^{*}=s^{*}$ where $s=$ $\bigwedge_{i \in \Gamma} r_{i}$. Since $B^{*} \in\left(\tau_{T}^{s^{*}}\right)^{*}=\tau_{\mathcal{M}_{R_{T}^{s}}}$, then $\mathcal{M}_{R_{T}^{s}}\left(B^{*}\right)=B=\mathcal{M}_{R_{T}^{r_{i}}}(A) \geq A^{*}$. So, $A \geq \mathcal{M}_{R_{T}^{s}}^{*}\left(B^{*}\right)=B^{*}$. Thus

$$
\mathcal{M}_{R_{T}^{s}}(A) \leq \mathcal{M}_{R_{T}^{s}}\left(\mathcal{M}_{R_{T}^{s}}^{*}\left(B^{*}\right)=\mathcal{M}_{R_{T}^{s}}\left(B^{*}\right)=B .\right.
$$

Since $s \leq r_{i}, \mathcal{M}_{R_{T}^{s}}(A) \geq \mathcal{M}_{R_{T}^{r_{i}}}(A)=B$. Thus $\mathcal{M}_{R_{T}^{s}}(A)=B$.

Theorem 3.3. Let $\mathbf{T}$ be an Alexandorv fuzzy topology on $X$. We have the following properties.

(1) Define $\mathbf{T}_{M_{T}}: L^{X} \rightarrow L$ as

$$
\mathbf{T}_{M_{T}}(A)=\bigvee\left\{r_{i}^{*} \in L \mid \mathcal{M}_{R_{T}^{r_{i}}}(A)=A^{*}\right\} .
$$

Then $\mathbf{T}_{M_{T}}=\mathbf{T}^{*}$ is an Alexandrov fuzzy topology on $X$.

(2) Define $\mathbf{T}_{M_{T^{*}}}: L^{X} \rightarrow L$ as

$$
\mathbf{T}_{M_{T^{*}}}(A)=\bigvee\left\{r_{i}^{*} \in L \mid \mathcal{M}_{R_{T}^{-r_{i}}}(A)=A^{*}\right\}
$$

Then $\mathbf{T}_{M_{T^{*}}}=\mathbf{T}$ is an Alexandrov fuzzy topology on $X$.

(3) $e_{L^{X}}\left(\mathcal{M}_{R_{T}^{r}}(A), B\right)=e_{L^{X}}\left(\mathcal{M}_{R_{T^{*}}^{r}}(B), A\right)$ for all $A, B \in L^{X}$.

(4) There exists an Alexandrov fuzzy topology $\mathbf{T}^{r}$ such that

$$
\mathbf{T}^{r}(A)=e_{L^{X}}\left(\mathcal{M}_{R_{T}^{r}}(A), A^{*}\right) .
$$


If $r \leq s$, then $\mathbf{T}^{r} \leq \mathbf{T}^{s}$ for all $A \in L^{X}$.

(5) There exists an Alexandrov fuzzy topology $\mathbf{T}^{* r}$ such that

$$
\mathbf{T}^{* r}(A)=e_{L^{X}}\left(\mathcal{M}_{R_{T}^{-r}}(A), A^{*}\right) .
$$

Moreover, $\mathbf{T}^{* r}(A)=\mathbf{T}^{r}\left(A^{*}\right)$ for all $A \in L^{X}$. If $r \leq s$, then $\mathbf{T}^{* r} \leq \mathbf{T}^{* s}$ for all $A \in L^{X}$.

(6) Define $\mathbf{T}_{M}: L^{X} \rightarrow L$ as

$$
\mathbf{T}_{M}(A)=\bigvee\left\{r^{*} \in L \mid \mathbf{T}^{r}(A)=\top\right\} .
$$

Then $\mathbf{T}_{M}=\mathbf{T}^{*}=\mathbf{T}_{M_{T}}$ is an Alexandrov fuzzy topology on $X$.

(7) Define $\mathbf{T}_{M^{*}}: L^{X} \rightarrow L$ as

$$
\mathbf{T}_{M^{*}}(A)=\bigvee\left\{r^{*} \in L \mid \mathbf{T}^{* r}(A)=\top\right\}
$$

Then $\mathbf{T}_{M^{*}}=\mathbf{T}=\mathbf{T}_{M_{T^{*}}}$ is an Alexandrov fuzzy topology on $X$.

Proof. (1) We only show that $\mathbf{T}_{M_{T}}=\mathbf{T}^{*}$. Let $\mathcal{M}_{R_{T}^{r_{i}}}(A)=A^{*}$. Then $A \in \tau_{\mathcal{M}_{R_{T}^{r_{i}}}}=$ $\left(\tau_{T}^{r *}\right)^{*}$ from Theorem 3.3.(6). So, $\mathbf{T}^{*}(A)=\mathbf{T}\left(A^{*}\right)=\mathbf{T}\left(\mathcal{M}_{R_{T}^{r_{i}}}(A)\right) \geq r_{i}^{*}$. Thus,

$$
\mathbf{T}_{M_{T}}(A)=\bigvee\left\{r_{i}^{*} \in L \mid \mathcal{M}_{R_{T}^{r_{i}}}(A)=A^{*}\right\} \leq \mathbf{T}^{*}(A) .
$$

Since $\mathbf{T}^{*}(A) \geq(\mathbf{T}(A))^{*}$, then $\tau_{T^{*}}^{s *}=\tau_{\mathcal{M}_{R_{T}^{s}}}$ with $s=\mathbf{T}(A)$. Thus,

$$
\mathbf{T}_{M_{T}}(A)=\bigvee\left\{r_{i}^{*} \in L \mid \mathcal{M}_{R_{T}^{r_{i}}}(A)=A^{*}\right\} \geq s^{*}=\mathbf{T}^{*}(A) .
$$

Hence $\mathbf{T}_{M_{T}}=\mathbf{T}^{*}$.

$$
\begin{aligned}
& e_{L^{X}}\left(\mathcal{M}_{R_{T}^{r}}(A), B\right)=\bigwedge_{y \in X}\left(\mathcal{M}_{R_{T}^{r}}(A)(y) \rightarrow B(y)\right) \\
& =\bigwedge_{y \in X}\left(\bigvee_{x \in X}\left(A^{*}(x) \odot R_{T}^{r}(x, y)\right) \rightarrow B(y)\right) \\
& =\bigwedge_{y \in X} \bigwedge_{x \in X}\left(R_{T}^{r}(x, y) \rightarrow\left(A^{*}(x) \rightarrow B(y)\right)\right) \\
& =\bigwedge_{y \in X} \bigwedge_{x \in X}\left(R_{T}^{r}(x, y) \rightarrow\left(B^{*}(y) \rightarrow A(x)\right)\right) \\
& =\bigwedge_{x \in X}\left(\bigvee_{y \in X}\left(B^{*}(y) \odot R_{T}^{r}(x, y)\right) \rightarrow A(x)\right) \\
& =e_{L^{X}}\left(\mathcal{M}_{R_{T}^{-r}}(B), A\right)
\end{aligned}
$$

(4) (T1) Since $\mathcal{M}_{R_{T}^{r}}\left(\alpha_{X}\right)(y)=\bigvee_{x \in X}\left(\alpha_{X}(x) \odot R_{T}^{r}(x, y)\right)=\alpha \odot \bigvee_{x \in X} R_{T}^{r}(x, y)=$ $\alpha, \mathbf{T}^{r}\left(\alpha_{X}\right)=e_{L^{X}}\left(\mathcal{M}_{R_{T}^{r}}\left(\alpha_{X}\right), \alpha_{X}\right)=\top$.

(T2) Since $\mathcal{M}_{R_{T}^{r}}\left(\bigvee_{i \in \Gamma} A_{i}\right) \leq \bigwedge_{i \in \Gamma} \mathcal{M}_{R_{T}^{r}}\left(A_{i}\right)$, we have

$$
\begin{aligned}
& \mathbf{T}^{r}\left(\bigvee_{i \in \Gamma} A_{i}\right)=e_{L^{X}}\left(\mathcal{M}_{R_{T}^{r}}\left(\bigvee_{i \in \Gamma} A_{i}\right),\left(\bigvee_{i \in \Gamma} A_{i}\right)^{*}\right) \\
& \geq e_{L^{X}}\left(\bigwedge_{i \in \Gamma} \mathcal{M}_{R_{T}^{r}}\left(A_{i}\right), \bigwedge_{i \in \Gamma} A_{i}^{*}\right) \\
& \geq \bigwedge_{i \in \Gamma} e_{L^{X}}\left(\mathcal{M}_{R_{T}^{r}}\left(A_{i}\right), A_{i}\right)=\bigwedge_{i \in \Gamma} \mathbf{T}^{r}\left(A_{i}\right)
\end{aligned}
$$




$$
\begin{aligned}
& \mathbf{T}^{r}\left(\bigwedge_{i \in \Gamma} A_{i}\right)=e_{L^{X}}\left(\mathcal{M}_{R_{T}^{r}}\left(\bigwedge_{i \in \Gamma} A_{i}\right),\left(\bigwedge_{i \in \Gamma} A_{i}\right)^{*}\right) \\
& =e_{L^{X}}\left(\bigvee_{i \in \Gamma} \mathcal{M}_{R_{T}^{r}}\left(A_{i}\right), \bigvee_{i \in \Gamma} A_{i}^{*}\right) \\
& \geq \bigwedge_{i \in \Gamma} e_{L^{X}}\left(\mathcal{M}_{R_{T}^{r}}\left(A_{i}\right), A_{i}^{*}\right)=\bigwedge_{i \in \Gamma} \mathbf{T}^{r}\left(A_{i}\right)
\end{aligned}
$$

(T3) Since $\alpha \odot \mathcal{M}_{R_{T}^{r}}(\alpha \odot A)=\mathcal{M}_{R_{T}^{r}}(\alpha \rightarrow(\alpha \odot A)) \leq \mathcal{M}_{R_{T}^{r}}(A)$, then $\mathcal{M}_{R_{T}^{r}}(\alpha \odot$ $A) \leq \alpha \rightarrow \mathcal{M}_{R_{T}^{r}}(A)$. Thus

$$
\begin{aligned}
& \mathbf{T}^{r}(\alpha \odot A)=e_{L^{X}}\left(\mathcal{M}_{R_{T}^{r}}(\alpha \odot A),(\alpha \odot A)^{*}\right) \\
& \geq e_{L^{X}}\left(\alpha \rightarrow \mathcal{M}_{R_{T}^{r}}(A), \alpha \rightarrow A^{*}\right) \\
& \geq e_{L^{X}}\left(\mathcal{M}_{R_{T}^{r}}(A), A^{*}\right)=\mathbf{T}^{r}(A)
\end{aligned}
$$

$$
\begin{aligned}
& \mathbf{T}^{r}(\alpha \rightarrow A)=e_{L^{X}}\left(\mathcal{M}_{R_{T}^{r}}(\alpha \rightarrow A),(\alpha \rightarrow A)^{*}\right) \\
& =e_{L^{X}}\left(\alpha \odot \mathcal{M}_{R_{T}^{r}}(A), \alpha \odot A^{*}\right) \\
& \geq e_{L^{X}}\left(\mathcal{M}_{R_{T}^{r}}(A), A^{*}\right)=\mathbf{T}^{r}(A)
\end{aligned}
$$

Hence $\mathbf{T}^{r}$ is an Alexandrov fuzzy topology. Since $\mathcal{M}_{R_{T}^{s}} \leq \mathcal{M}_{R_{T}^{r}}$ for $r \leq s, \mathbf{T}^{s}(A)=$ $e_{L^{X}}\left(\mathcal{M}_{R_{T}^{s}}(A), A^{*}\right) \geq e_{L^{X}}\left(\mathcal{M}_{R_{T}^{r}}(A), A^{*}\right)=\mathbf{T}^{r}(A)$.

(5) From a similar method as (4), $\mathbf{T}^{* r}$ is an Alexandrov fuzzy topology. By (3), $\mathbf{T}^{r}\left(A^{*}\right)=e_{L^{X}}\left(\mathcal{M}_{R_{T}^{r}}\left(A^{*}\right), A\right)=e_{L^{X}}\left(\mathcal{M}_{R_{T^{*}}^{r}}(A), A^{*}\right)=\mathbf{T}^{* r}(A)$ for all $A \in L^{X}$.

(6) Since $\mathbf{T}^{r}(A)=e_{L^{X}}\left(\mathcal{M}_{R_{T}^{r}}(A), A^{*}\right)=\top$ iff $A^{*}=\mathcal{M}_{R_{T}^{r}}(A)$, by $(9)$,

$$
\begin{aligned}
\mathbf{T}_{M}(A) & =\bigvee\left\{r^{*} \in L \mid \mathbf{T}^{r}(A)=\top\right\} \\
& =\bigvee\left\{r^{*} \in L \mid \mathcal{M}_{R_{T}^{r}}^{r}(A)=A^{*}\right\} \\
& =\mathbf{T}_{M_{T}}(A)=\mathbf{T}^{*}(A) .
\end{aligned}
$$

(2) and (7) are similarly proved as (1) and (6), respectively.

Example 3.4. Let $\left(L=[0,1], \odot, \rightarrow,^{*}\right)$ be a complete residuated lattice with a strong negation.

(1) Let $X=\{x, y, z\}$ be a set. Define a map $\mathbf{T}:[0,1]^{X} \rightarrow[0,1]$ as

$$
\mathbf{T}(A)=A(x) \rightarrow A(z) .
$$

Trivially, $\mathbf{T}\left(\alpha_{X}\right)=1$

Since $\alpha \odot A(x) \rightarrow \alpha \odot A(z) \geq A(x) \rightarrow A(z)$ from Lemma $2.4(14), \mathbf{T}(\alpha \odot$ $A) \geq \mathbf{T}(A)$. Since $(\alpha \rightarrow A(x)) \rightarrow(\alpha \rightarrow A(z)) \geq A(x) \rightarrow A(z)$ from Lemma $2.4(10), \mathbf{T}(\alpha \rightarrow A) \geq \mathbf{T}(A)$. By Lemma $2.4(8), \mathbf{T}\left(\bigvee_{i \in \Gamma} A_{i}\right) \geq \bigwedge_{i \in \Gamma} \mathbf{T}\left(A_{i}\right)$ and $\mathbf{T}\left(\bigwedge_{i \in \Gamma} A_{i}\right) \geq \bigwedge_{i \in \Gamma} \mathbf{T}\left(A_{i}\right)$. Hence $\mathbf{T}$ is an Alexandrov fuzzy topology.

If $\mathbf{T}(A)=A(x) \rightarrow A(z) \geq r^{*}$, then $A(z) \geq A(x) \odot r^{*}$. Put $A(x)=1, A(y)=0$. So, $R_{T}^{r}(x, y)=\bigwedge\left\{A(x) \rightarrow A(y) \mid \mathbf{T}(A) \geq r^{*}\right\}=0$ and $R_{T}^{r}(x, z)=\bigwedge\{A(x) \rightarrow A(z) \mid$ 
$\left.\mathbf{T}(A) \geq r^{*}\right\}=r^{*}$, similarly, we can obtain

$$
\left(\begin{array}{ccc}
R_{T}^{r}(x, x)=1 & R_{T}^{r}(x, y)=0 & R_{T}^{r}(x, z)=r^{*} \\
R_{T}^{r}(y, x)=0 & R_{T}^{r}(y, y)=1 & R_{T}^{r}(y, z)=0 \\
R_{T}^{r}(z, x)=0 & R_{T}^{r}(z, y)=0 & R_{T}^{r}(z, z)=1
\end{array}\right)
$$

By Theorem 3.2(3), we obtain $\mathcal{M}_{R_{T}^{r}}(A)(y)=\bigvee_{x \in X}\left(A^{*}(x) \odot R_{T}^{r}(x, y)\right)$ such that

$$
\mathcal{M}_{R_{T}^{r}}(A)=\left(A^{*}(x), A^{*}(y), A^{*}(z) \vee\left(A^{*}(x) \odot r^{*}\right)\right)
$$

If $A^{*}(x) \odot r^{*} \leq A^{*}(z)$, then $\mathcal{M}_{R_{T}^{r}}(A)=A^{*}$. Thus $A \in \tau_{\mathcal{M}_{R_{T}^{r}}}$. Moreover, since $\mathbf{T}^{*}(A)=A^{*}(x) \rightarrow A^{*}(z) \geq r^{*}$ iff $A^{*}(z) \geq A^{*}(x) \odot r^{*}, A \in \tau_{T^{*}}^{r^{*}}$ iff $A \in \tau_{\mathcal{M}_{R_{T}^{r}}}$. So, $\tau_{T^{*}}^{r^{*}}=\tau_{\mathcal{M}_{R_{T}^{r}}}$. From Theorem 3.3(1), we have

$$
\begin{aligned}
\mathbf{T}_{M_{T}}(A) & =\bigvee\left\{r^{*} \in L \mid \mathcal{M}_{R_{T}^{r}}(A)=A\right\} \\
& =A^{*}(x) \rightarrow A^{*}(z)=\mathbf{T}\left(A^{*}\right)=\mathbf{T}^{*}(A)
\end{aligned}
$$

Moreover, we obtain

$$
\begin{aligned}
\mathbf{T}^{r}(A)= & \bigwedge_{x \in X}\left(\mathcal{M}_{R_{T}^{r}}(A)(x) \rightarrow A^{*}(x)\right) \\
= & \left(A^{*}(x) \odot r^{*}\right) \rightarrow A^{*}(z)=r^{*} \rightarrow\left(A^{*}(x) \rightarrow A^{*}(z)\right) . \\
& \mathbf{T}_{M}(A)=\bigvee\left\{r^{*} \in L \mid \mathbf{T}^{r}(A)=1\right\} \\
= & A^{*}(x) \rightarrow A^{*}(z) .
\end{aligned}
$$

Hence $\mathbf{T}_{M}=\mathbf{T}_{M_{T}}=\mathbf{T}^{*}$.

$$
\mathcal{M}_{R_{T}^{r}}\left(1_{x}^{*}\right)(z)=\bigwedge\left\{B(z) \mid B \geq 1_{x}, \mathbf{T}(B) \geq r^{*}\right\}
$$

Since $B(x)=1$ and $\mathbf{T}(B)=1 \rightarrow B(z)=B(z) \geq r^{*}$, then $\mathcal{M}_{R_{T}^{r}}\left(1_{x}^{*}\right)(z)=r^{*}$.

$$
\begin{gathered}
\mathcal{M}_{R_{T}^{r}}\left(1_{x}^{*}\right)(x)=\bigwedge\left\{B(x) \mid B \geq 1_{x}, \mathbf{T}(B) \geq r^{*}\right\}=1 \\
\mathcal{M}_{R_{T}^{r}}\left(1_{x}^{*}\right)(y)=\bigwedge\left\{B(y) \mid B \geq 1_{x}, \mathbf{T}(B) \geq r^{*}\right\}=0 \\
\mathcal{M}_{R_{T}^{r}}\left(1_{z}^{*}\right)(x)=\bigwedge\left\{B(x) \mid B \geq 1_{z}, \mathbf{T}(B) \geq r^{*}\right\}
\end{gathered}
$$

Since $B(z)=1$ and $\mathbf{T}(B)=B(x) \rightarrow 1=1$, then $\mathcal{M}_{R_{T}^{r}}\left(1_{z}^{*}\right)(x)=0$.

$$
\left(\begin{array}{ccc}
\mathcal{M}_{R_{T}^{r}}\left(1_{x}^{*}\right)(x)=1 & \mathcal{M}_{R_{T}^{r}}\left(1_{x}^{*}\right)(y)=0 & \mathcal{M}_{R_{T}^{r}}\left(1_{x}^{*}\right)(z)=r^{*} \\
\mathcal{M}_{R_{T}^{r}}\left(1_{y}^{*}\right)(x)=0 & \mathcal{M}_{R_{T}^{r}}\left(1_{y}^{*}\right)(y)=1 & \mathcal{M}_{R_{T}^{r}}\left(1_{y}^{*}\right)(z)=0 \\
\mathcal{M}_{R_{T}^{r}}\left(1_{z}^{*}\right)(x)=0 & \mathcal{M}_{R_{T}^{r}\left(1_{z}^{*}\right)(y)=0} & \mathcal{M}_{R_{T}^{r}}\left(1_{z}^{*}\right)(z)=1
\end{array}\right)
$$

Then $R_{T}^{r}(x, y)=\mathcal{M}_{R_{T}^{r}}\left(1_{x}^{*}\right)(y)$.

(2) By (1), we obtain a map $\mathbf{T}^{*}:[0,1]^{Y} \rightarrow[0,1]$ as

$$
\mathbf{T}^{*}(A)=A^{*}(x) \rightarrow A^{*}(z)=A(z) \rightarrow A(x) .
$$


Since $\mathbf{T}^{*}(A)=A(z) \rightarrow A(x) \geq r^{*}$, then $A(x) \geq A(z) \odot r^{*}$. Put $A(z)=1, A(y)=0$. So, $R_{T^{*}}^{r}(z, y)=\bigwedge\left\{A(z) \rightarrow A(y) \mid \mathbf{T}^{*}(A) \geq r^{*}\right\}=0$ and $R_{T^{*}}^{r}(z, x)=\bigwedge\{A(z) \rightarrow$ $\left.A(x) \mid \mathbf{T}(A) \geq r^{*}\right\}=r^{*}$

$$
\left(\begin{array}{ccc}
R_{T^{*}}^{r}(x, x)=1 & R_{T^{*}}^{r}(x, y)=0 & R_{T^{*}}^{r}(x, z)=0 \\
R_{T^{*}}^{r}(y, x)=0 & R_{T^{*}}^{r}(y, y)=1 & R_{T^{*}}^{r}(y, z)=0 \\
R_{T^{*}}^{r}(z, x)=r^{*} & R_{T^{*}}^{r}(z, y)=0 & R_{T^{*}}^{r}(z, z)=1
\end{array}\right)
$$

Moreover, $R_{T^{*}}^{r}(x, y)=R_{T}^{-r}(x, y)=R_{T}^{r}(y, x)$ for all $x, y \in X$.

$$
\begin{gathered}
\mathcal{M}_{R_{T^{*}}^{r}}(A)(y)=\bigvee_{x \in X}\left(A(x) \odot R_{T *}^{r}(x, y)\right) \\
\mathcal{M}_{R_{T^{*}}^{r}}(A)=\left(A^{*}(x) \vee\left(A^{*}(z) \odot r^{*}\right), A^{*}(y), A^{*}(z)\right)
\end{gathered}
$$

If $A^{*}(z) \odot r^{*} \leq A^{*}(x)$, then $\mathcal{M}_{R_{T^{*}}^{r}}(A)=A^{*}$. If $\mathcal{M}_{R_{T^{*}}^{r}}(A)=A^{*}$, then $A^{*}(z) \odot r^{*} \leq$ $A^{*}(x)$. Moreover, since $\mathbf{T}(A)=A(x) \rightarrow A(z) \geq r^{*}$ iff $A^{*}(z) \odot r^{*} \leq A^{*}(z), A \in \tau_{T}^{r^{*}}$ iff $A \in \tau_{\mathcal{M}_{R_{T^{*}}^{r}}}$. Thus

$$
\begin{aligned}
\mathbf{T}_{M_{T^{*}}}(A) & =\bigvee\left\{r^{*} \in L \mid \mathcal{M}_{R_{T^{*}}^{r}}(A)=A\right\} \\
& =A^{*}(z) \rightarrow A^{*}(x)=\mathbf{T}(A)=A(x) \rightarrow A(z) .
\end{aligned}
$$

Moreover, we obtain

$$
\begin{aligned}
\mathbf{T}^{* r}(A)= & \bigwedge_{x \in X}\left(\mathcal{M}_{R_{T}^{r}}(A)(x) \rightarrow A(x)\right) \\
= & \left(A^{*}(z) \odot r^{*}\right) \rightarrow A^{*}(x)=r^{*} \rightarrow\left(A^{*}(z) \rightarrow A^{*}(x)\right) . \\
& \mathbf{T}_{M^{*}}(A)= \\
& =\bigvee\left\{r^{*} \in L \mid \mathbf{T}^{* r}(A)=1\right\} \\
& =A^{*}(z) \rightarrow A^{*}(x)=\mathbf{T}(A) .
\end{aligned}
$$

Hence $\mathbf{T}_{M^{*}}=\mathbf{T}_{M_{T^{*}}}=\mathbf{T}$.

$$
\mathcal{M}_{R_{T^{*}}^{r}}\left(1_{x}^{*}\right)(z)=\bigwedge\left\{B(z) \mid B \geq 1_{x}, \mathbf{T}^{*}(B) \geq r^{*}\right\}
$$

Since $B(x)=1$ and $\mathbf{T}^{*}(B)=B(z) \rightarrow 1=1$, then $\mathcal{M}_{R_{T^{*}}^{r}}\left(1_{x}^{*}\right)(z)=0$.

$$
\begin{gathered}
\mathcal{M}_{R_{T^{*}}^{r}}\left(1_{z}^{*}\right)(y)=\bigwedge\left\{B(y) \in L^{X} \mid B \geq 1_{z}, \mathbf{T}^{*}(B) \geq r^{*}\right\}=0 \\
\mathcal{M}_{R_{T^{*}}^{r}}\left(1_{y}^{*}\right)(y)=\bigwedge\left\{B(y) \in L^{X} \mid B \geq 1_{y}, \mathbf{T}^{*}(B) \geq r^{*}\right\}=1 \\
\mathcal{M}_{R_{T^{*}}^{r}}\left(1_{z}^{*}\right)(x)=\bigwedge\left\{B(x) \in L^{X} \mid B \geq 1_{z}, \mathbf{T}^{*}(B) \geq r^{*}\right\}
\end{gathered}
$$

Since $B(z)=1$ and $\mathbf{T}^{*}(B)=1 \rightarrow B(x)=B(x) \geq r^{*}$, then $B(x) \geq r^{*}$. We have $\mathcal{M}_{R_{T^{*}}^{r}}\left(1_{z}^{*}\right)(x)=r^{*}$.

$$
\left(\begin{array}{ccc}
\mathcal{M}_{R_{T^{*}}^{r}}\left(1_{x}^{*}\right)(x)=1 & \mathcal{M}_{R_{T^{*}}^{r}}\left(1_{x}^{*}\right)(y)=0 & \mathcal{M}_{R_{T^{*}}^{r}}\left(1_{x}^{*}\right)(z)=0 \\
\mathcal{M}_{R_{T^{*}}^{r}}\left(1_{y}^{*}\right)(x)=0 & \mathcal{M}_{R_{T^{*}}^{r}}\left(1_{y}^{*}\right)(y)=1 & \mathcal{M}_{R_{T^{*}}^{r}}\left(1_{y}^{*}\right)(z)=0 \\
\mathcal{M}_{R_{T^{*}}^{r}}\left(1_{z}^{*}\right)(x)=r^{*} & \mathcal{M}_{R_{T^{*}}^{r}}\left(1_{z}^{*}\right)(y)=0 & \mathcal{M}_{R_{T^{*}}^{r}}\left(1_{z}^{*}\right)(z)=1
\end{array}\right)
$$


Then $R_{T^{*}}^{r}(x, y)=\mathcal{M}_{R_{T^{*}}^{r}}\left(1_{x}^{*}\right)(y)$.

(3) Let $\left(L=[0,1], \odot, \rightarrow{ }^{*}\right)$ be a complete residuated lattice with a strong negation defined by, for each $n \in N$,

$$
x \odot y=\left(\left(x^{n}+y^{n}-1\right) \vee 0\right)^{\frac{1}{n}}, x \rightarrow y=\left(1-x^{n}+y^{n}\right)^{\frac{1}{n}} \wedge 1, x^{*}=\left(1-x^{n}\right)^{\frac{1}{n}} .
$$

By (1) and (2), we obtain

$$
\begin{aligned}
\mathbf{T}(A)= & \left(1-A(x)^{n}+A(z)^{n}\right)^{\frac{1}{n}} \wedge 1, \mathbf{T}^{*}(A)=\left(1-A(z)^{n}+A(x)^{n}\right)^{\frac{1}{n}} \wedge 1 . \\
& R_{T}^{r}=\left(\begin{array}{ccc}
1 & 0 & \left(1-r^{n}\right)^{\frac{1}{n}} \\
0 & 1 & 0 \\
0 & 0 & 1
\end{array}\right) R_{T^{*}}^{r}=\left(\begin{array}{ccc}
1 & 0 & 0 \\
0 & 1 & 0 \\
\left(1-r^{n}\right)^{\frac{1}{n}} & 0 & 1
\end{array}\right) \\
& \mathcal{M}_{R_{T}^{r}}(A)=\left(A^{*}(x), A^{*}(y), A^{*}(z) \vee\left(\left(\left(A^{*}(x)\right)^{n}-r^{n}\right) \vee 0\right)^{\frac{1}{n}}\right) \\
& \mathcal{M}_{R_{T^{*}}^{r}}(A)=\left(A^{*}(x) \vee\left(\left(\left(A^{*}(z)\right)^{n}-r^{n}\right) \vee 0\right)^{\frac{1}{n}}, A^{*}(y), A^{*}(z)\right)
\end{aligned}
$$

Since $\mathbf{T}(A)=\left(1-A(x)^{n}+A(z)^{n}\right)^{\frac{1}{n}} \wedge 1 \geq\left(1-x^{n}\right)^{\frac{1}{n}}$, we have

$$
\begin{aligned}
& \tau_{T}^{r^{*}}=\tau_{\mathcal{M}_{R^{*}}}=\left\{A \in L^{X} \mid A^{n}(x)-A^{n}(z) \leq r^{n}\right\} \\
& \tau_{T^{*}}^{r^{*}}=\tau_{\mathcal{M}_{R_{T}^{r}}}=\left\{A \in L^{X} \mid A^{n}(z)-A^{n}(x) \leq r^{n}\right\} . \\
& \mathbf{T}^{r}(A) \quad=\left(A^{*}(x) \odot r^{*}\right) \rightarrow A^{*}(z)=\left(r^{n}-\left(A^{*}(x)\right)^{n}+\left(A^{*}(z)\right)^{n}\right)^{\frac{1}{n}} \wedge 1 \\
& \mathbf{T}^{* r}(A)=\left(A(z) \odot r^{*}\right) \rightarrow A(x)=\left(r^{n}-\left(A^{*}(z)\right)^{n}+\left(A^{*}(x)\right)^{n}\right)^{\frac{1}{n}} \wedge 1 .
\end{aligned}
$$

\section{REFERENCES}

1. R. Bělohlávek: Fuzzy Relational Systems. Kluwer Academic Publishers, New York, 2002.

2. P. Hájek: Metamathematices of Fuzzy Logic. Kluwer Academic Publishers, Dordrecht, 1998.

3. U. Höhle \& S.E. Rodabaugh: Mathematics of Fuzzy Sets: Logic, Topology, and Measure Theory, The Handbooks of Fuzzy Sets Series 3. Kluwer Academic Publishers, Boston, 1999.

4. Fang Jinming: I-fuzzy Alexandrov topologies and specialization orders. Fuzzy Sets and Systems 158 (2007), 2359-2374.

5. Y.C. Kim: Alexandrov $L$-topologies and $L$-join meet approximation operators. International Journal of Pure and Applied Mathematics. 91 (2014), no. 1, 113-129.

6. H. Lai \& D. Zhang: Fuzzy preorder and fuzzy topology. Fuzzy Sets and Systems $\mathbf{1 5 7}$ (2006), 1865-1885.

7. __ Concept lattices of fuzzy contexts: Formal concept analysis vs. rough set theory. Int. J. Approx. Reasoning 50 (2009), 695-707.

8. Z. Pawlak: Rough sets. Int. J. Comput. Inf. Sci. 11 (1982), 341-356. 
9. ___ Rough probability. Bull. Pol. Acad. Sci. Math. 32 (1984), 607-615.

10. A.M. Radzikowska \& E.E. Kerre: A comparative study of fuzy rough sets. Fuzzy Sets and Systems 126 (2002), 137-155.

11. Y.H. She \& G.J. Wang: An axiomatic approach of fuzzy rough sets based on residuated lattices. Computers and Mathematics with Applications 58 (2009), 189-201.

12. Zhen Ming Ma \& Bao Qing Hu: Topological and lattice structures of L-fuzzy rough set determined by lower and upper sets. Information Sciences 218 (2013), 194-204.

Department of Mathematics, Gangneung-Wonju National University, Gangneung 210702, KoreA

Email address: yck@gwnu.ac.kr 